\title{
Dynamic control of chaotic resonators
}

\author{
Di Falco A. ${ }^{a}$, Bruck R. ${ }^{\mathrm{b}}$, Liu C. ${ }^{\mathrm{c}}$, Muskens O. ${ }^{\mathrm{b}}$, and Fratalocchi A. ${ }^{\mathrm{c}}$ \\ ${ }^{a}$ SUPA, School of Physics and Astronomy, University of St Andrews, North Haugh, St \\ Andrews KY16 9SS, UK \\ ${ }^{\mathrm{b}}$ School of Physics and Astronomy, Faculty of Physical Sciences and Engineering, University of \\ Southampton, Southampton SO17 1BJ, UK \\ 'PRIMALIGHT, Faculty of Electrical Engineering; Applied Mathematics and Computational \\ Science, King Abdullah University of Science and Technology (KAUST), Thuwal 23955-6900, \\ Saudi Arabia
}

\begin{abstract}
We report on the all-optical control of chaotic optical resonators based on silicon on insulator (SOI) platform. We show that simple non-chaotic cavities can be tuned to exhibit chaotic behavior via intense optical pumping, inducing a local change of refractive index. To this extent we have fabricated a number of devices and demonstrated experimentally and theoretically that chaos can be triggered on demand on an optical chip.
\end{abstract}

Keywords: Chaotic resonators, All-optical control of chaos, Photonic Crystals, Photonic crystal resonators, Nonlinear optics

\section{INTRODUCTION}

Photonic Crystals $(\mathrm{PhC})$ have a prominent role in modern integrated optics, because of the extreme design versatility, mediated by the dispersion control and the existence of bandgaps. ${ }^{1-4}$ We recently used this versatility to fabricate and study chaotic optical resonators. ${ }^{5,6}$ We have shown that the eigenvalues of a chaotic cavity tend to have the same value, and that this effect can be exploited for broadband energy storage. ${ }^{7}$ We have also shown that losses play a non trivial role in chaotic dynamics, and can lead to localized states for light in time and space, akin to rogue waves. ${ }^{8}$ In these studies, the effect are controlled with careful choice of the topology, for example arranging the holes to form a uniform billiard cavity, or by using coupling waveguides of different width. Here we show that the degree of chaos supported by a microcavity can be controlled dynamically, using light itself. For this experiment, we a square (non-chaotic) cavity obtained on Silicon on Insulator. We then pump from above the cavity with pulsed blue light and collect the transmission of the probe pulse in the near infrared, for different pump-probe delays. Since the pump is focused on a small region of the cavity, our study permits to assess the effect of pumping on different regions of the resonator. The analysis of these activity maps demonstrates that nonlinear optics can be used to control reversibly the chaotic behavior of light in optical resonators.

\section{FABRICATION OF PHOTONIC CRYSTAL RESONATORS}

The chaotic resonators were fabricated in standard SOI technology, with a silicon top layer of $220 \mathrm{~nm}$, capping a $\mathrm{SiO}_{2}$ buffer layer with a thickness of $2 \mu \mathrm{m}$, which is thick enough to prevent light from being radiated into the silicon substrate. The high contrast between the top silicon and the oxide enables single mode guiding for light in the $1550 \mathrm{~nm}$ region. The photonic crystal resonator is obtained via standard electron beam lihtography techniques. The sample was cleaned with acetone and isopropanol and rinsed in deionised water. A $350 \mathrm{~nm}$ thick film of a positive tone photoresist (ZEP) was deposited via spinning at $3250 \mathrm{rpm}$ and baked for $10 \mathrm{~min}$ at $180{ }^{\circ} \mathrm{C}$. The sample was then irradiated with electrons accelerated at $30 \mathrm{KV}$ with a Raith E-line electron beam lithography system, with a nominal dose of $55 \mu \mathrm{C} / \mathrm{cm}^{2}$. The film was then developed for $45 s$ in xylene

Further author information: (Send correspondence to A.D.F)

A.D.F.: E-mail: adf10@st-andrews.ac.uk, Telephone: +44 1334463165

Quantum Sensing and Nano Electronics and Photonics XIII, edited by Manijeh Razeghi,

Gail J. Brown, Jay S. Lewis, Proc. of SPIE Vol. 9755, 97551F · @ 2016 SPIE

CCC code: $0277-786 \mathrm{X} / 16 / \$ 18 \cdot$ doi: $10.1117 / 12.2217762$

Proc. of SPIE Vol. $975597551 \mathrm{~F}-1$ 


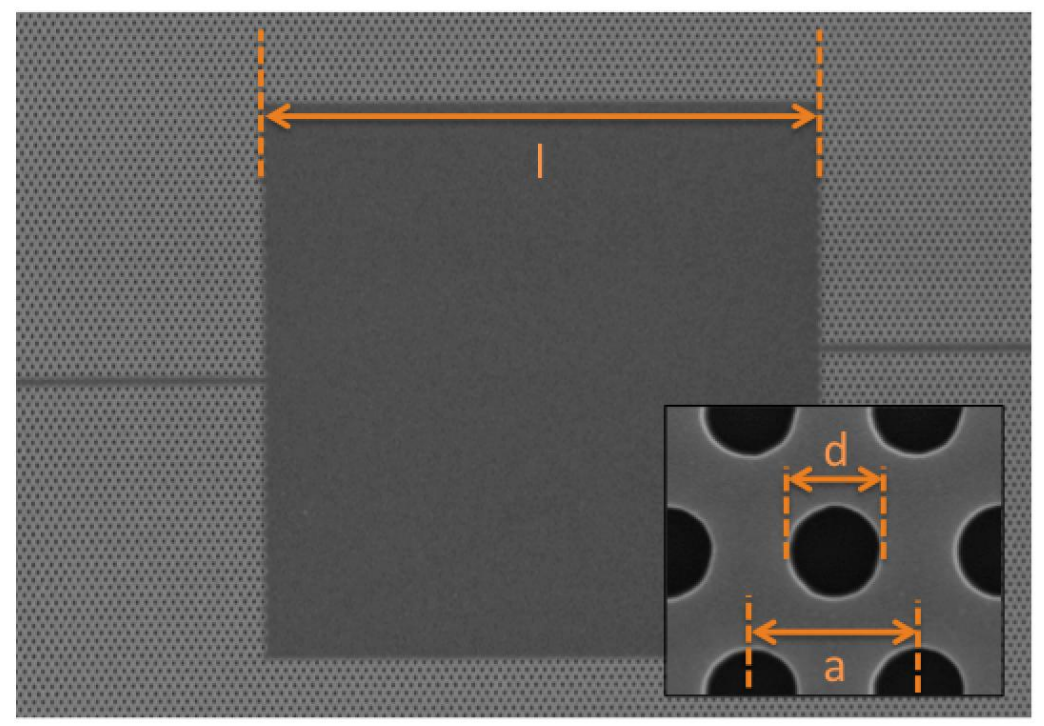

Figure 1. SEM picture of a typical classical $\mathrm{PhC}$ resonator, with side $l=20 \mu \mathrm{m}$, and input and output coupling waveguides. The inset shows a blown up region of the $\mathrm{PhC}$ lattice, and highlights its geometrical parameters.

at $23{ }^{\circ} \mathrm{C}$, in a gentle ultrasonic bath, removing the exposed resist. The features were then transferred onto the silicon layer via reactive ion etching, using a custom made system, using a $50: 50$ blend of $S F_{6}$ and $C H F_{3}$ gases, at a pressure of $5 \times 10^{-3}$ mbar for $2 \mathrm{~min}$, with a DC bias of $200 \mathrm{~V}$. The residual resist was removed from the sample with a 5 min long exposure to UV light, followed by an ultrasonic bath in trichloroethylene, for as long as required for complete removal. The sample was finally cleaved for end-fire coupling and mounted on a stage for the optical characterisation. Fig. 1 shows a scanning electron microscope (SEM) picture of a typical resonator. The $\mathrm{PhC}$ resonator is defined by surrounding a square region, with side $l=20 \mu m$, with an array of air holes etched in the silicon. The geometrical parameters of the triangular PhC arrangement (lattice constant $a=450 \mathrm{~nm}$ and holes diameter $d=300 \mathrm{~nm}$, see inset of fig. 1) were selected to create a bandgap of several hundreds nanometers around the $1550 \mathrm{~nm}$ region of the optical spectrum, for the TE polarisation (with the electric field parallel to the $\mathrm{PhC}$ plane). Light was coupled to the cavity area and out of it via defect $\mathrm{PhC}$ waveguides, obtained removing a single row of holes. The vertical position of the in- and out-coupling waveguides was misaligned to avoid enforcing undesired preferential coupling symmetries.

\section{SECTION EXPERIMENTAL SETUP}

The sample was measured with a pump and probe scheme, ${ }^{9-13}$ using light emitted from a tunable optical parametric amplifier (OPA), pumped by a Ti:sapphire laser, as sketched in fig. 2. The system generates $150 f s$ pulses with a repetition rate of $250 \mathrm{kHz}$. Pulses at wavelength $\lambda=400 \mathrm{~nm}$ were used to pump the cavity from above. Pulses in the range of $\lambda=1550 \mathrm{~nm}$, with TE polarization, were launched on the coupling waveguides and collected at the output with microscope objectives. The pump pulses, focused by a microscope objective on the surface of the resonator, generate free carriers that modulate locally the complex refractive index of the material. This modulation perturbs the uniform distribution of permittivity of the resonator, promoting a chaotic behavior for the pulses at the probe frequencies. Crucially, the time delay between the pump and probe pulses can be tuned well beyond the lifetime of the induced carriers. Hence, the tuning of the behavior can be traced without ambiguity. Additionally, the pump pulse can be focused down to a spot size of $2 \mu m$, and scanned across the whole area of interest with a piezoelectric actuator, with an accuracy of $100 \mathrm{~nm}$. Scanning the wavelength of the probe pulses, and acquiring the outcoupled light for different excitation parameters, gives a full picture of the behavior of the chaotic resonator. For large $|\Delta t|$ one expects to record a non chaotic dynamics, since the probe 

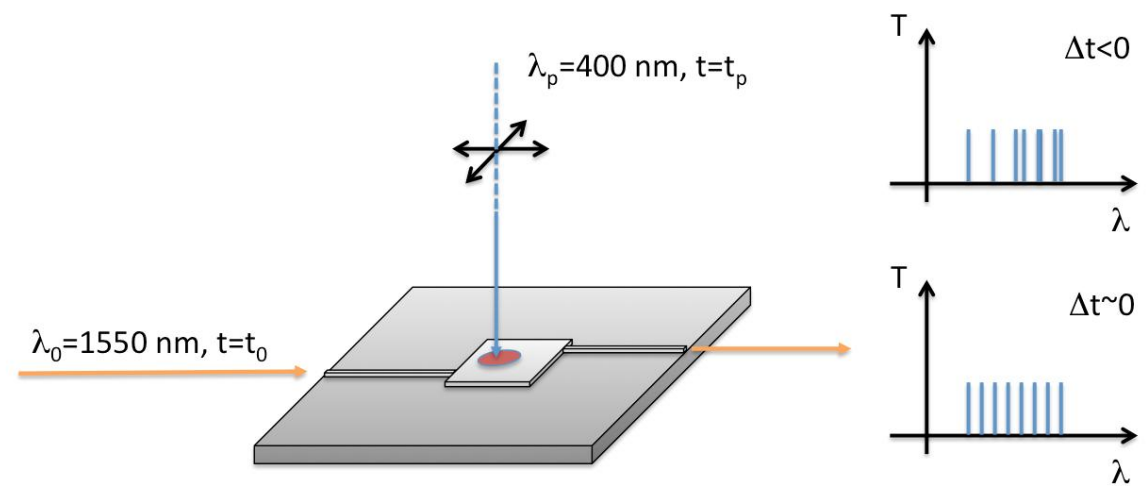

$$
\Delta t=t_{0}-t_{p}
$$

Pulses duration: $150 \mathrm{fs}$

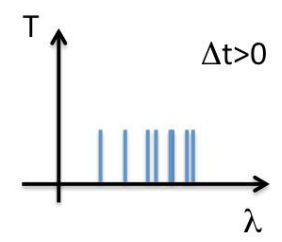

Figure 2. Schematic of the experimental setup. Pulses of $150 \mathrm{fs}$ in the near infrared and at $400 \mathrm{~nm}$, generated from an OPA, are used to probe and pump, respectively, the photonic microcavity. The delay between the pump and probe pulses can be tuned.

pulses propagate in non perturbed cavity. For small positive $\Delta t$, the probe pulses is expected to experience strong perturbations, which could lead to a chaotic dynamics.

\section{RESULTS AND DISCUSSIONS}

Fig. 3 shows a typical scan of the experimental parameters. The pump is focused on a low symmetry point inside the cavity and for different wavelength of the probe, the delay between pump and probe is varied. A convenient figure of merit to analyse the perturbation of the cavity is the chance of transmission, expressed as $\Delta T / T$. As expected, for $\Delta t<<0$, when the probe pulse enters the cavity well before the pump pulse, the dynamics is essentially unperturbed. For $\Delta t \geq 0$ the situations is markedly different. The transmission vs $\lambda$ is strongly modified. Monitoring the evolution of $\Delta T / T$ in time, coupled to the analysis of the power density spectrum, gives direct access to the transition from regular to chaotic behavior.

Pumping the cavity in different points gives a direct information on how critical is a given region in the determining the propagation of the pulse in the resonator. Fig. 4 shows a typical activity map for the square $\mathrm{PhC}$ resonator. This is obtained integrating over a large range of probe wavelengths and times the normalised transmission. The result give a compelling overview of the full dynamics. Trivially, pumping outside the cavity area (identified with a dash line in the figure), produce no change in the transmission. Pumping the access and exit waveguides has instead a very strong effect. Inside the cavity, it is easy to recognise that the direct path from the input to the output waveguide is a region of high activity. This tells us that despite the careful misalignment of the feeding channels, there exist a preferential coupling trajectory for the photons. The most interesting features are the high activity regions out with the central area, which confirms that the behavior of light in the $\mathrm{PhC}$ resonator is strongly affected by the perturbation.

A combination of the information extracted from figs. 3 and 4 gives full account of the transition for regular to chaotic dynamics in our resonators, and the relative importance that different regions of the cavity play in the light dynamics. A possible way of assessing the degree of chaos in this class of resonators is to count the number and position of the supported resonant modes. While the range of probe wavelengths used is fairly large, the number of modes supported by the cavity is not large enough to create a convincingly clear statistics. For this reason, as a figure of merit, we used the spectral rigidity $\left(\Delta_{3}\right)$, which accounts for the correlation between 


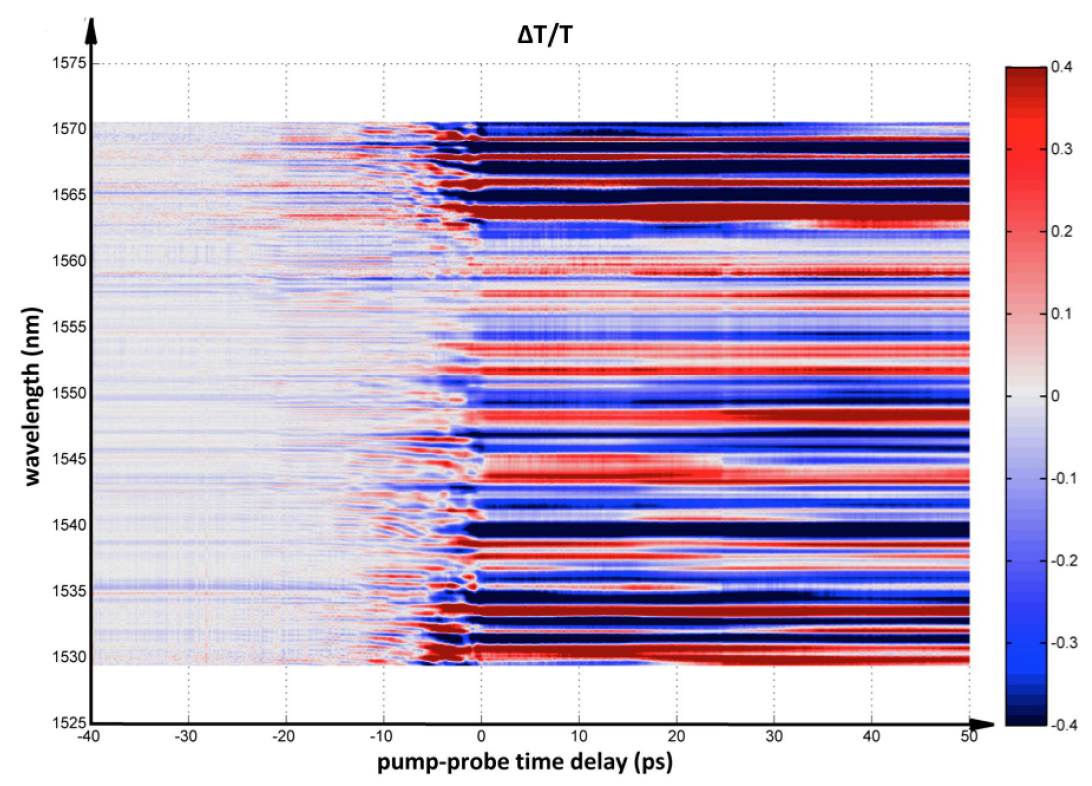

Figure 3. Normalized change of transmission as function of probe wavelength and pump/probe delay.

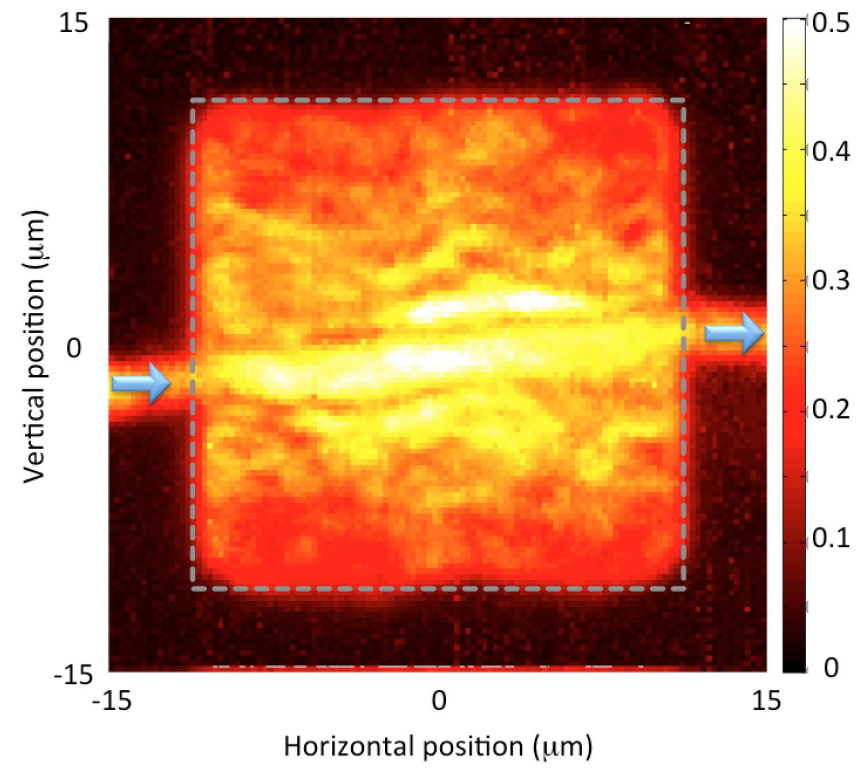

Figure 4. Activity map of the $\mathrm{PhC}$ resonator, in terms respect to the position of the pumping spot.

resonances, and is related to the degree of disorder of a given system. ${ }^{14}$ For a given number of modes (energy levels), the smaller $\Delta_{3}$, the higher the degree of chaos. Fig. 5 shows $\Delta_{3}$ vs the number of found modes, for the point of interest of fig. 3, and compares it with a fully chaotic behavior (red curve) and a non chaotic distribution of modes (black curve), for different delay values, thus demonstrating the onset of chaotic dynamics for large $\Delta t$.

In conclusion we have demonstrated the dynamic tuning of the chaotic behavior of an integrated photonic cavities, using a nonlinear all optical pumping scheme. 


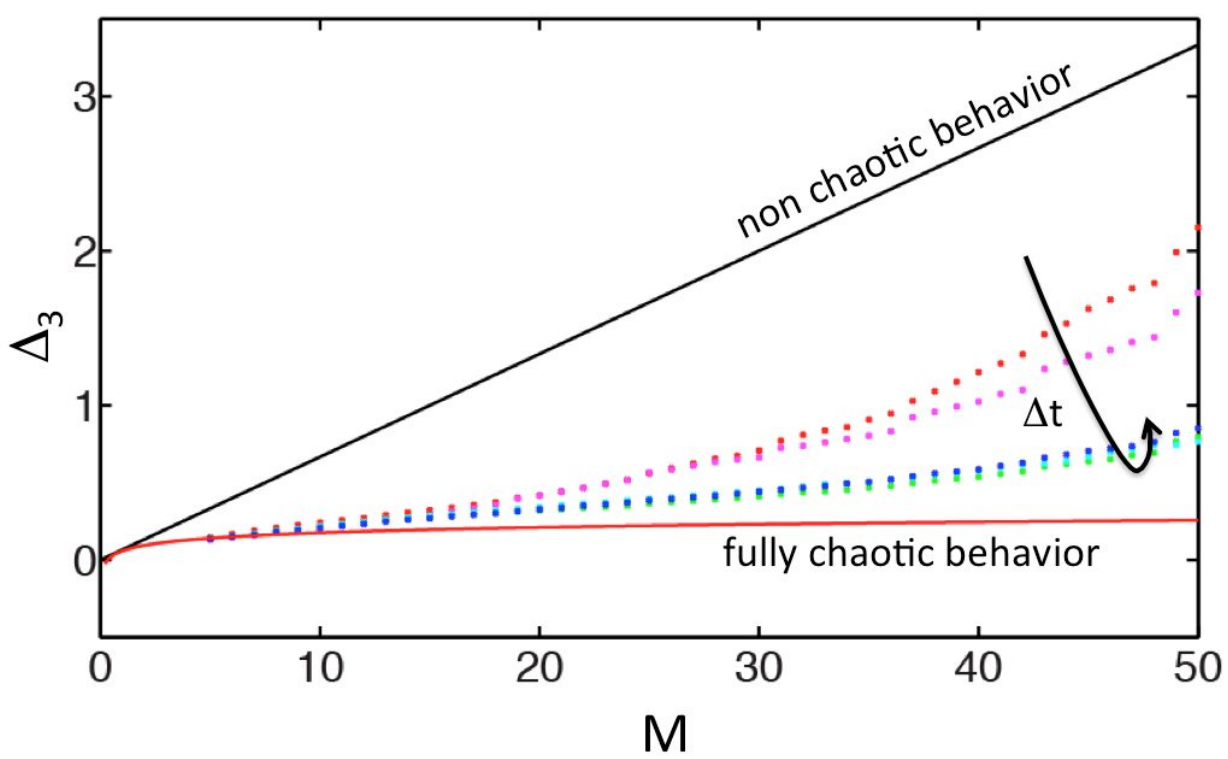

Figure 5. Spectral rigidity in normalised units vs the number of modes supported by the cavity (M). Different symbols corresponds to different time delays.

\section{ACKNOWLEDGMENTS}

OM acknowledges support from EPSRC through grant no. EP/J016918.

\section{REFERENCES}

[1] Conti, C., Di Falco, A., and Assanto, G., "Frequency generation within the forbidden band gap: All optical Rabi-like splitting in photonic crystals and microcavities," Physical Review E 70, 066614 (Dec. 2004).

[2] Conti, C., Di Falco, A., and Assanto, G., "Controlled transmission in the forbidden photonic bandgap via transient nonlinear states," Optics Letters 29(24), 2902-2904 (2004).

[3] Conti, C., Di Falco, A., and Assanto, G., "Optical parametric oscillations in isotropic photonic crystals," Optics Express 12(5), 823-828 (2004).

[4] Di Falco, A., Conti, C., and Assanto, G., "Photonic crystal wires for optical parametric oscillators in isotropic media," Applied Physics B-Lasers And Optics 79(1), 9-13 (2004).

[5] Di Falco, A., Krauss, T., and Fratalocchi, A., "Lifetime statistics of quantum chaos studied by a multiscale analysis," Applied Physics Letters 100(18), 184101 (2012).

[6] Liu, C., Di Falco, A., and Fratalocchi, A., "Dicke phase transition with multiple superradiant states in quantum chaotic resonators," Physical review X 4(2), 021048 (2014).

[7] Liu, C., Di Falco, A., Molinari, D., Khan, Y., Ooi, B., Krauss, T., and Fratalocchi, A., "Enhanced energy storage in chaotic optical resonators," Nature Photonics 7(6), 473-478 (2013).

[8] Liu, C., van der Wel, R. E. C., Rotenberg, N., Kuipers, L., Krauss, T. F., Di Falco, A., and Fratalocchi, A., "Triggering extreme events at the nanoscale in photonic seas," Nature Physics 11, 358-363 (Mar. 2015).

[9] Bruck, R., Mills, B., Troia, B., Thomson, D. J., Gardes, F. Y., Hu, Y., Mashanovich, G. Z., Passaro, V. M., Reed, G. T., and Muskens, O. L., "Device-level characterization of the flow of light in integrated photonic circuits using ultrafast photomodulation spectroscopy," Nature Photonics 9(1), 54-60 (2015).

[10] Bruck, R., Mills, B., Thomson, D. J., Troia, B., Passaro, V., Mashanovich, G. Z., Reed, G. T., and Muskens, O. L., "Picosecond optically reconfigurable filters exploiting full free spectral range tuning of single ring and vernier effect resonators," Optics express 23(9), 12468-12477 (2015). 
[11] Strudley, T., Bruck, R., Mills, B., and Muskens, O. L., "An ultrafast reconfigurable nanophotonic switch using wavefront shaping of light in a nonlinear nanomaterial," Light: Science 85 Applications 3(9), e207 (2014).

[12] Abb, M., Bakkers, E. P., and Muskens, O. L., "Ultrafast dephasing of light in strongly scattering gap nanowires," Physical review letters 106(14), 143902 (2011).

[13] Muskens, O. L., Venn, P., van der Beek, T., and Wellens, T., "Partial nonlinear reciprocity breaking through ultrafast dynamics in a random photonic medium," Physical review letters 108(22), 223906 (2012).

[14] Dyson, F. J. and Mehta, M. L., "Statistical theory of the energy levels of complex systems. iv," Journal of Mathematical Physics 4(5), 701-712 (1963). 